\title{
MODELAGEM MATEMÁTICA PARA A DESCRIÇÃO DO PROCESSO DE SECAGEM DO FEIJÃO (Phaseolus vulgaris L.) EM CẢMADAS DELGADAS
}

\author{
PAULO C. CORRÊA ${ }^{1}$, OSVALDO RESENDE ${ }^{2}$, ANA P. MARTINAZZO ${ }^{3}$, \\ ANDRÉ L. D. GONELI ${ }^{4}$, FERNANDO M. BOTELHO ${ }^{5}$
}

\begin{abstract}
RESUMO: O objetivo do presente trabalho foi a obtenção e a avaliação das curvas de secagem do feijão (Phaseolus vulgaris L.) e ajustar diferentes modelos matemáticos aos valores experimentais do teor de água para diversas condições do ar. Foram utilizados grãos de feijão colhidos com teor de água de 0,92 (b.s.) e submetidos à secagem até o teor 0,14 (b.s.) sob condições controladas de temperatura $\left(35 ; 45\right.$ e $\left.55^{\circ} \mathrm{C}\right)$ e umidade relativa do ar de secagem de $40 \pm 2 \%$. Aos dados experimentais, foram ajustados 12 modelos matemáticos citados na literatura específica e utilizados para a representação do processo de secagem de produtos agrícolas. Pelos resultados obtidos e baseando-se em parâmetros estatísticos, pode-se concluir que metade dos modelos testados representa bem o fenômeno de secagem do feijão. Dentre esses, o modelo clássico de Page foi selecionado, pela sua simplicidade e pelo seu uso disseminado no meio científico, para descrever a cinética de secagem dos produtos vegetais. A relação entre a constante de secagem $\mathrm{k}$ desse modelo e a temperatura do ar pode ser descrita pela relação de Arrhenius, apresentando energia de ativação de $10,08 \mathrm{~kJ} \mathrm{~mol}^{-1}$.
\end{abstract}

PALAVRAS-CHAVE: feijão, modelagem matemática, teor de água.

\section{MATHEMATICAL MODELLING FOR DESCRIBING THE DRYING PROCESS OF THE EDIBLE BEAN (Phaseolus vulgaris L.) IN THIN LAYERS}

\begin{abstract}
The objective of this work was to obtain and to evaluate the drying curves of edible bean (Phaseolus vulgaris L.) and to fit different mathematical models to different experimental data of moisture content and drying air conditions. Edible beans harvested at moisture content of 0.92 (d.b.) were dried until achieving the moisture content of 0.14 (d.b.) under controlled drying air temperatures $\left(35 ; 45\right.$ and $55^{\circ} \mathrm{C}$ ) and relative humidity of $40 \pm 2 \%$. Twelve mathematical models cited in the literature were fitted to the experimental data in order to represent the drying process. According to the results and based on the statistical parameters it can be concluded that half of the tested models represent well the edible bean drying phenomena. Within them the classic Page model was selected due to its simplicity and widely use within researchers to describe the agricultural product drying kinetic. The relationship between the drying constant $\mathrm{k}$ and the drying air temperature can be described through Arrhenius's equation presenting activation energy of $10.08 \mathrm{~kJ} \mathrm{~mol}^{-1}$.
\end{abstract}

KEYWORDS: edible bean, mathematical model, moisture content.

\footnotetext{
${ }^{1}$ Eng $^{\mathrm{o}}$ Agrônomo, Prof. Adjunto, Departamento de Engenharia Agrícola, UFV, Viçosa - MG , Fone: (0XX31) 3891.2270, copace@ufv.br

${ }^{2}$ Eng $^{\mathrm{O}}$ Agrícola, Prof. Adjunto, Departamento de Agronomia, UNIR, Rolim de Moura - RO, osvresende@ yahoo.com.br

${ }^{3}$ Eng ${ }^{0}$ Agrícola, Doutoranda em Engenharia Agrícola, UFV, Viçosa - MG. Bolsista CNPq.

${ }^{4}$ Eng $^{\mathrm{O}}$ Agrônomo, M.Sc., Doutorando em Engenharia Agrícola, UFV, Viçosa - MG. Bolsista CNPq.

${ }^{5}$ Bolsista Iniciação Científica CNPq, UFV, Viçosa - MG.

Recebido pelo Conselho Editorial em: 30-6-2005
}

Aprovado pelo Conselho Editorial em: 14-6-2007 


\section{INTRODUÇÃO}

A secagem dos produtos é o processo mais utilizado para assegurar sua qualidade e sua estabilidade, considerando que a diminuição da quantidade de água do material reduz a atividade biológica e as mudanças químicas e físicas que ocorrem durante o armazenamento.

O fenômeno de redução do teor de água de grãos envolve, simultaneamente, a transferência de calor e massa, que podem alterar de forma substancial a qualidade e as propriedades físicas do produto, dependendo do método e das condições de secagem (HALL, 1980).

O processo de secagem de um produto pode ser dividido em período de velocidade constante e outro de velocidade decrescente. Durante o período de velocidade constante, a temperatura do produto mantém-se igual à do ar de secagem saturado, e as transferências de calor e massa compensam-se, ou seja, o ar recupera, sob a forma de vapor, o que perdeu sob a forma de calor. No período de velocidade decrescente de secagem, a taxa de transporte interno de água é menor do que a taxa de evaporação; dessa forma, a transferência de calor do ar para o produto não é compensada pela transferência do vapor de água e, conseqüentemente, a temperatura do grão aumenta, tendendo a atingir a temperatura do ar de secagem (BROOKER et al., 1992).

No desenvolvimento e no aperfeiçoamento de equipamentos utilizados para a secagem de grãos, é de fundamental importância a simulação e a obtenção de informações teóricas a respeito do comportamento de cada produto durante a remoção de água. Para a simulação, cujo princípio se fundamenta na secagem de sucessivas camadas delgadas do produto, utiliza-se modelo matemático que representa, satisfatoriamente, sua perda de água durante o período de secagem (BERBET et al., 1995).

As curvas de secagem em camada delgada variam com a espécie, variedade, condições ambientais, métodos de preparo pós-colheita, entre outros fatores. Nesse sentido, diversos modelos matemáticos têm sido utilizados para descrever o processo de secagem de produtos agrícolas.

Embora vários modelos tenham sido propostos para predizer o comportamento da secagem de grãos e sementes, na maioria das vezes, as relações semi-empíricas e empíricas têm-se mostrado como melhores opções para predizer o processo de secagem, apesar de sua validade estar restrita às condições sob as quais os dados experimentais foram obtidos (BROOKER et al., 1992).

Esses modelos, geralmente, baseiam-se em variáveis externas ao produto, como a temperatura e a umidade relativa do ar de secagem. Entretanto, não fornecem indicações sobre os fenômenos de transporte de energia e de água no interior dos grãos e consideram que todo o processo de secagem ocorre somente no período de taxa decrescente.

As equações semi-empíricas têm como base a lei de resfriamento de Newton para transferência de calor por convecção, presumindo-se que, durante a secagem, as condições sejam isotérmicas e que a transferência de água seja restrita à superfície do produto (INCROPERA \& DEWITT, 1992).

Recentemente, têm sido realizados inúmeros trabalhos com o objetivo de identificar as características de diversos produtos agrícolas durante a secagem, como: trigo (SUN \& WOODS, 1994), feijão-preto (AFONSO JÚNIOR \& CORRÊA, 1999), arroz em casca (BASUNIA \& ABE, 2001), uva (YALDIZ et al., 2001; RAMOS et al., 2004; RAMOS et al., 2005), pimenta (KAYMAK-ERTEKIN, 2002; AKPINAR et al., 2003), pêra (LAHSASNI et al., 2004) e trigo parbolizado (MOHAPATRA \& RAO, 2005), dentre outros. Durante a modelagem e a simulação dos processos de secagem de produtos agrícolas, diversos trabalhos correlacionaram satisfatoriamente os coeficientes dos modelos ajustados aos parâmetros de secagem, principalmente a temperatura, a umidade relativa e a vazão do ar (MADAMBA et al., 1996; AFONSO JÚNIOR \& CORRÊA, 1999; OZDEMIR \& DERVES, 1999; AZZOUZ et al., 2002; KROKIDA et al., 2003; KROKIDA et al., 2004; MOHAPATRA \& RAO, 2005). 
Considerando a importância do feijão na alimentação brasileira e a escassez de informações teóricas a respeito dos fenômenos que ocorrem durante a secagem, para suas diversas variedades, o presente trabalho teve como objetivos estimar e avaliar as curvas de secagem do feijão (Phaseolus vulgaris L.) e ajustar diferentes modelos matemáticos aos valores experimentais para diversas condições do ar.

\section{MATERIAL E MÉTODOS}

O presente trabalho foi desenvolvido no Laboratório de Propriedades Físicas e Qualidade de Produtos Agrícolas, pertencente ao Centro Nacional de Treinamento em Armazenagem (CENTREINAR), localizado na Universidade Federal de Viçosa, Viçosa - MG.

Para a condução do experimento, foram utilizados grãos de feijão do grupo vermelho, colhidos manualmente, com teor de água de aproximadamente 0,92 (b.s.). Os teores de água do produto foram determinados pelo método da estufa, $105 \pm 1^{\circ} \mathrm{C}$, até massa constante.

A secagem do produto foi realizada para condições controladas de temperatura $(35 ; 45$ e $55^{\circ} \mathrm{C}$ ) e umidade relativa do ar de secagem de $40 \pm 2 \%$.

As três condições de temperatura e umidade relativa do ar foram fornecidas por meio de unidade condicionadora de atmosfera, de fabricação da empresa Aminco, modelo Aminco-Aire 150/300 CFM.

Foram colocadas no interior do equipamento bandejas removíveis de metal com fundo telado para permitir a passagem do ar através da massa de produto, contendo cada uma, inicialmente, $50 \mathrm{~g}$ de feijão, em duas repetições. A quantidade de produto utilizada promoveu o completo preenchimento da bandeja, de forma a assegurar a camada delgada de produto. $\mathrm{O}$ fluxo de ar foi monitorado com o auxílio de um anemômetro de fio quente e mantido constante em torno de $4 \mathrm{~m}^{3} \mathrm{~s}^{-1} \mathrm{~m}^{-2}$. A temperatura e a umidade relativa do ar foram monitoradas por meio de psicrômetro instalado próximo às bandejas, contendo as amostras.

Durante o processo de secagem, as bandejas com as amostras foram pesadas periodicamente. Utilizou-se de balança digital da empresa Marte, modelo AS 2000, de 0,01 g de resolução. O tempo entre as leituras foi controlado por meio da diferença de massas (conhecendo-se o teor de água inicial), de forma a não se permitirem diferenças grandes de teor de água entre as leituras. Para fins de modelagem matemática, considerou-se a secagem do feijão até que o produto atingisse o teor de água de 0,14 (b.s.), aproximadamente, estabelecido como ponto final da secagem. Depois disso, o produto permaneceu nas bandejas até que atingisse seu teor de água de equilíbrio com a condição do ar especificada.

Para a determinação das razões de umidade do feijão durante a secagem, nas diferentes condições de ar, utilizou-se da eq.(1):

$$
\mathrm{RU}=\frac{\mathrm{U}^{*}-\mathrm{U}_{\mathrm{e}}^{*}}{\mathrm{U}_{\mathrm{i}}^{*}-\mathrm{U}_{\mathrm{e}}^{*}}
$$

em que,

RU - razão de umidade do produto, adimensional;

$\mathrm{U}^{*}$ - teor de água do produto, decimal b.s.;

$\mathrm{U}_{\mathrm{i}}^{*}$ - teor de água inicial do produto, decimal b.s., e

$\mathrm{U}_{\mathrm{e}}^{*}$ - teor de água de equilíbrio do produto, decimal b.s.

Aos dados experimentais da secagem do feijão, foram ajustados os modelos matemáticos freqüentemente utilizados para a representação da secagem de produtos agrícolas (AFONSO JÚNIOR \& CORRÊA, 1999; AKPINAR et al., 2003; ERTEKIN \& YALDIZ, 2004; LAHSASNI et al., 2004), cujas expressões estão apresentadas na Tabela 1. 
TABELA 1. Modelos matemáticos utilizados para predizer o fenômeno de secagem de produtos agrícolas.

\begin{tabular}{|c|c|c|}
\hline Designação do Modelo & Modelo & \\
\hline $\mathrm{RU}=1+\mathrm{at}+\mathrm{bt}^{2}$ & Wang e Singh & (2) \\
\hline$R U=a \exp (-k t)+(1-a) \exp \left(-k_{1} t\right)$ & Verna & (3) \\
\hline$R U=\exp \left(\left(-a-\left(a^{2}+4 b t\right)^{0,5}\right) / 2 b\right.$ & Thompson & (4) \\
\hline $\mathrm{RU}=\exp \left(-\mathrm{k} \mathrm{t^{ \textrm {n } }}\right)$ & Page & (5) \\
\hline $\mathrm{RU}=\exp (-\mathrm{kt})$ & Newton & (6) \\
\hline $\mathrm{RU}=\mathrm{a} \exp \left(-\mathrm{kt}^{\mathrm{n}}\right)+\mathrm{bt}$ & Midilli & (7) \\
\hline $\mathrm{RU}=\mathrm{a} \exp (-\mathrm{kt})+\mathrm{c}$ & Logarítmico & (8) \\
\hline$R U=a \exp (-k t)$ & Henderson e Pabis & (9) \\
\hline$R U=a \exp (-k t)+b \exp \left(-k_{o} t\right)+c \exp \left(-k_{1} t\right)$ & Henderson e Pabis Modificado & $(10)$ \\
\hline$R U=a \exp (-k t)+(1-a) \exp (-k a t)$ & Exponencial de Dois Termos & (11) \\
\hline$R U=a \exp \left(-k_{o} t\right)+b \exp \left(-k_{1} t\right)$ & Dois Termos & (12) \\
\hline$R U=a \exp (-k t)+(1-a) \exp (-k b t)$ & Aproximação da Difusão & $(13)$ \\
\hline
\end{tabular}

em que,

RU - razão de umidade do produto, adimensional;

$\mathrm{t}$ - tempo de secagem, $\mathrm{h}$;

$\mathrm{k}, \mathrm{k}_{\mathrm{o}}, \mathrm{k}_{1}$ - constantes de secagem, $\mathrm{h}^{-1}$, e

$\mathrm{a}, \mathrm{b}, \mathrm{c}, \mathrm{n}$ - coeficientes dos modelos.

Para avaliar a influência da temperatura nos coeficientes dos modelos ajustados à secagem do feijão, foi utilizada a equação de Arrhenius [eq.(14)]:

$$
k=d \exp \left(\frac{-E_{a}}{R T_{a}}\right)
$$

em que,

d - fator pré-exponencial;

$\mathrm{E}_{\mathrm{a}}$ - energia de ativação, $\mathrm{J} \mathrm{mol}^{-1}$;

$\mathrm{R}$ - constante universal dos gases, $8,314 \mathrm{~J} \mathrm{~mol}^{-1} \mathrm{~K}^{-1}$, e

$\mathrm{T}_{\mathrm{a}}$ - temperatura absoluta, $\mathrm{K}$.

Para o ajuste dos modelos matemáticos, foi realizada análise de regressão linear e não-linear, pelo método Gauss Newton, utilizando-se do "software" STATISTICA 5.0 ${ }^{\circledR}$. O grau de ajuste de cada modelo considerou a significância do coeficiente de regressão pelo teste t, adotando o nível de $1 \%$ de probabilidade, a magnitude do coeficiente de determinação $\left(\mathrm{R}^{2}\right)$, a magnitude do erro médio relativo $(\mathrm{P})$ e do erro médio estimado (SE) e a verificação do comportamento da distribuição dos resíduos. O erro médio relativo e o erro médio estimado, para cada um dos modelos, foram calculados conforme eqs.(15) e (16):

$$
\begin{aligned}
& P=\frac{100}{n} \sum \frac{|Y-\hat{Y}|}{Y} \\
& S E=\sqrt{\frac{\sum(Y-\hat{Y})^{2}}{G L R}}
\end{aligned}
$$

em que,

Y - valor observado experimentalmente;

$\hat{Y}$ - valor calculado pelo modelo;

$\mathrm{N}$ - número de observações experimentais, e

GLR - graus de liberdade do modelo (número de observações menos o número de parâmetros do modelo). 


\section{RESULTADOS E DISCUSSÃO}

Os valores experimentais do teor de água de equilíbrio para os grãos de feijão, nas condições utilizadas neste experimento, foram de 0,108; 0,097 e 0,087, respectivamente, para as temperaturas de $35 ; 45$ e $55^{\circ} \mathrm{C}$.

Na Figura 1, estão apresentados os valores experimentais da secagem de feijão realizada em diversas condições de temperatura e na umidade relativa do ar de $40 \pm 2 \%$. Observa-se que não foi possível detectar o período de taxa de secagem constante para as condições testadas, mesmo considerando o alto teor de água inicial do produto $(0,92$ b.s.). A taxa de secagem acentua-se com o aumento da temperatura numa mesma umidade relativa, concordando com os inúmeros resultados relatados na literatura referente a esse tema de investigação.

De acordo com KREYGER (1973), grãos e sementes, em geral, apresentam o período de secagem à taxa constante muito curto, ou inexistente, porque, nas condições operacionais de secagem, as resistências às transferências de água encontram-se essencialmente no seu interior, tornando a taxa de evaporação superficial acentuadamente superior à taxa de reposição de água do interior para a superfície do produto.

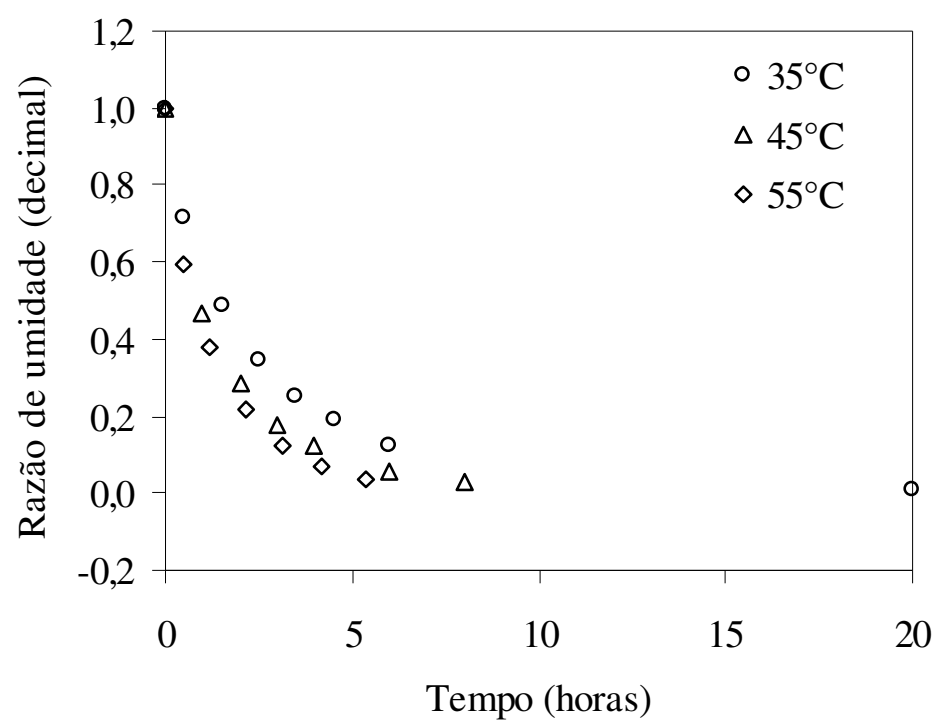

FIGURA 1. Valores experimentais da razão de umidade (decimal) e do tempo de secagem (horas) para as condições de temperatura $\left(35 ; 45\right.$ e $\left.55^{\circ} \mathrm{C}\right)$ e umidade relativa do ar de $40 \pm 2 \%$, durante a secagem do feijão.

Na Tabela 2, encontram-se os parâmetros estatísticos utilizados para a comparação entre os 12 modelos de secagem analisados, nas diversas condições de secagem utilizadas para o feijão.

Para as três combinações de temperatura e umidade relativa utilizadas na secagem do feijão, observa-se que os modelos matemáticos ajustados aos dados experimentais apresentaram coeficientes de determinação $\left(\mathrm{R}^{2}\right)$ superior ou próximo a $98 \%$ (Tabela 3), indicando, de acordo com MADAMBA et al. (1996), representação satisfatória do fenômeno em estudo, exceto para os modelos Wang e Singh (2) e Verna (3). Para as condições analisadas, os modelos Page (5), Midilli (7), Henderson e Pabis Modificado (10), Dois Termos (12) e Aproximação da Difusão (13) apresentaram menores valores do erro médio estimado, e magnitudes para o erro médio relativo inferior ou ligeiramente superior a 10\%, indicando, de acordo com MOHAPATRA \& RAO (2005), serem adequados para a descrição do fenômeno. Verifica-se, ainda, que esses modelos obtiveram, para a maioria das condições de secagem, distribuição aleatória dos resíduos, resultando, assim, em ajustes satisfatórios aos dados experimentais da secagem do feijão em camada fina e sob as condições experimentadas. 
TABELA 2. Coeficientes de determinação $\left(\mathrm{R}^{2}\right)$, erros médio relativo $(\mathrm{P})$ e estimado (SE), e tendência de distribuição dos resíduos para os 12 modelos analisados durante a secagem do feijão, nas diversas condições de temperatura $\left({ }^{\circ} \mathrm{C}\right)$ e umidade relativa do ar de $40 \pm 2 \%$.

\begin{tabular}{|c|c|c|c|c|}
\hline \multicolumn{5}{|c|}{ Temperatura $35^{\circ} \mathrm{C}$} \\
\hline Modelo & $\mathrm{R}^{2}(\%)$ & SE (decimal) & $\mathrm{P}(\%)$ & $\begin{array}{l}\text { Distribuição dos } \\
\text { Resíduos }\end{array}$ \\
\hline Wang e Singh & 83,98 & 0,143 & 63,15 & Tendenciosa \\
\hline Verna & 99,97 & 0,006 & 10,41 & Aleatória \\
\hline Thompson & 99,85 & 0,013 & 28,45 & Tendenciosa \\
\hline Page & 99,97 & 0,005 & 4,89 & Aleatória \\
\hline Newton & 98,00 & 0,047 & 23,72 & Tendenciosa \\
\hline Midilli & 99,97 & 0,005 & 3,24 & Aleatória \\
\hline Logaritmico & 98,83 & 0,042 & 90,56 & Tendenciosa \\
\hline Henderson e Pabis & 98,57 & 0,043 & 21,11 & Tendenciosa \\
\hline Henderson e Pabis Modificado & 99,99 & 0,004 & 0,54 & Aleatória \\
\hline Exponencial de Dois Termos & 99,58 & 0,023 & 15,59 & Tendenciosa \\
\hline Dois Termos & 99,97 & 0,007 & 10,41 & Aleatória \\
\hline Aproximação da Difusão & 99,97 & 0,006 & 10,41 & Aleatória \\
\hline \multicolumn{5}{|c|}{ Temperatura $45^{\circ} \mathrm{C}$} \\
\hline Wang e Singh & 89,11 & 0,123 & 83,97 & Tendenciosa \\
\hline Verna & 99,99 & 0,004 & 2,79 & Aleatória \\
\hline Thompson & 99,99 & 0,004 & 3,15 & Tendenciosa \\
\hline Page & 99,98 & 0,002 & 1,50 & Tendenciosa \\
\hline Newton & 98,92 & 0,038 & 29,65 & Tendenciosa \\
\hline Midilli & 99,99 & 0,001 & 0,54 & Aleatória \\
\hline Logaritmico & 99,58 & 0,027 & 18,09 & Tendenciosa \\
\hline Henderson e Pabis & 98,86 & 0,040 & 28,55 & Tendenciosa \\
\hline Henderson e Pabis Modificado & 99,99 & 0,001 & 0,29 & Aleatória \\
\hline Exponencial de Dois Termos & 99,76 & 0,018 & 15,66 & Tendenciosa \\
\hline Dois Termos & 99,99 & 0,004 & 2,79 & Aleatória \\
\hline Aproximação da Difusão & 99,99 & 0,004 & 2,79 & Aleatória \\
\hline \multicolumn{5}{|c|}{ Temperatura $55^{\circ} \mathrm{C}$} \\
\hline Wang e Singh & 91,84 & 0,109 & 60,17 & Tendenciosa \\
\hline Verna & 61,15 & 0,260 & 131,60 & Tendenciosa \\
\hline Thompson & 98,53 & 0,046 & 25,20 & Tendenciosa \\
\hline Page & 99,96 & 0,007 & 5,13 & Aleatória \\
\hline Newton & 98,53 & 0,042 & 25,19 & Tendenciosa \\
\hline Midilli & 99,99 & 0,004 & 3,43 & Tendenciosa \\
\hline Logaritmico & 99,35 & 0,034 & 17,03 & Tendenciosa \\
\hline Henderson e Pabis & 98,77 & 0,042 & 22,08 & Tendenciosa \\
\hline Henderson e Pabis Modificado & 99,99 & 0,003 & 1,46 & Tendenciosa \\
\hline Exponencial de Dois Termos & 99,80 & 0,017 & 8,82 & Tendenciosa \\
\hline Dois Termos & 99,99 & 0,002 & 1,48 & Tendenciosa \\
\hline Aproximação da Difusão & 99,99 & 0,002 & 1,48 & Tendenciosa \\
\hline
\end{tabular}

Dessa forma, a seleção e a recomendação do melhor modelo fundamentaram-se na simplicidade e no número de coeficientes. Dentre os modelos que apresentaram bons ajustes aos dados experimentais, o modelo de Page foi selecionado para representar o fenômeno de secagem do feijão devido a sua simplicidade de aplicação e por ser, tradicionalmente, recomendado e aplicado para predizer o fenômeno de secagem de diversos produtos agrícolas (RAMOS et al., 
1993/1994; CHRIST, 1996), incluindo sementes de feijão-preto (AFONSO JÚNIOR \& CORRÊA, 1999).

Na Tabela 3, estão os coeficientes do modelo de Page ajustados para a secagem do feijão em diferentes condições de temperatura e umidade relativa do ar.

TABELA 3. Coeficientes ajustados para o modelo de Page em diferentes condições de temperatura $\left(35 ; 45\right.$ e $\left.55^{\circ} \mathrm{C}\right)$ e umidade relativa do ar de $40 \pm 2 \%$, durante a secagem do feijão.

\begin{tabular}{clll}
\hline Temperatura $\left({ }^{\circ} \mathrm{C}\right)$ & \multicolumn{1}{c}{35} & \multicolumn{1}{c}{45} & \multicolumn{1}{c}{55} \\
\hline $\mathrm{k}$ & $0,00207^{* * *}$ & $0,00182^{* *}$ & $0,00162^{* *}$ \\
$\mathrm{n}$ & $0,665^{* *}$ & $0,737^{* *}$ & $0,768^{* *}$ \\
\hline
\end{tabular}

${ }^{* *}$ Significativo a $1 \%$ de probabilidade, pelo teste $\mathrm{t}$.

Analisando os resultados, observa-se que a magnitude da constante de secagem (k) para o modelo de Page, que representa o efeito das condições externas de secagem, diminuiu com a elevação da temperatura do ar de secagem. Segundo MADAMBA et al. (1996) e BABALIS \& BELESSIOTIS (2004), a constante de secagem $(\mathrm{k})$ pode ser utilizada como aproximação para caracterizar o efeito da temperatura e está relacionada à difusividade efetiva no processo de secagem no período decrescente e à difusão líquida que controla o processo.

Na Figura 2, é apresentada a influência da temperatura na constante de secagem (K), ajustada pelo modelo de Page, descrita por meio da representação de Arrhenius.

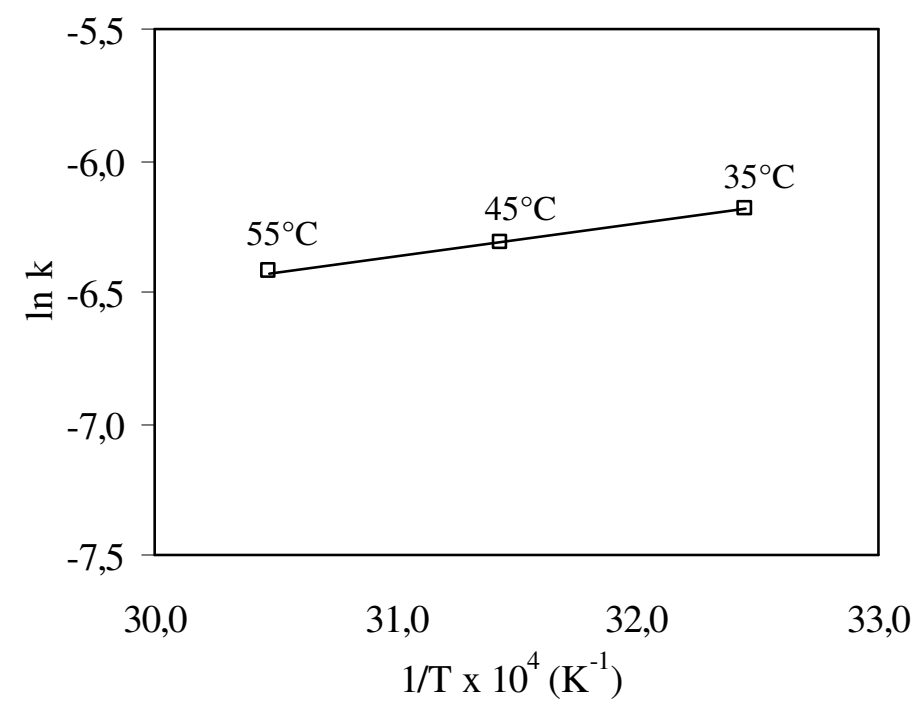

FIGURA 2. Representação de Arrhenius para a constante de secagem k do modelo de Page durante a secagem do feijão em camada fina.

A linearidade mostrada na Figura 2 evidencia a uniformidade ou a constância da variação da taxa de secagem dentro da faixa de temperatura estudada. A eq.(17) representa o coeficiente da expressão de Arrhenius, ajustado para o coeficiente k do modelo de Page, na secagem do feijão em camada fina.

$$
\mathrm{k}=3,70210^{-5} \exp \left(\frac{10083,7}{\mathrm{R} \mathrm{T}_{\mathrm{a}}}\right)
$$

Observa-se que a energia de ativação para o fenômeno de secagem do feijão foi de $10,08 \mathrm{~kJ} \mathrm{~mol}^{-1}$ para a faixa de temperatura entre 35 e $55^{\circ} \mathrm{C}$. Termodinamicamente, a energia de ativação é definida, como a facilidade com que as moléculas de água superam a barreira de energia durante a migração no interior do produto. Nos processos de secagem, quanto menor a energia de ativação, maior será a difusividade de água no produto. ZOGZAS et al. (1996) ressaltam que a 
energia de ativação para produtos agrícolas varia entre 12,7 a $110 \mathrm{~kJ} \mathrm{~mol}^{-1}$; entretanto, para o presente trabalho, a energia de ativação foi ligeiramente inferior, provavelmente devido ao alto teor de água inicial. Na literatura especializada, são encontrados valores da energia de ativação para diversos produtos agrícolas. DOYMAZ \& PALA (2003) observaram que, para o milho, a energia de ativação era da ordem de $29,56 \mathrm{~kJ} \mathrm{~mol}^{-1}$, para a temperatura entre 55 e $75{ }^{\circ} \mathrm{C}$; RAMESH (2003) verificou valor de $36,4 \mathrm{~kJ} \mathrm{~mol}^{-1}$ para o arroz cozido, para a faixa de temperatura de 110 a $180{ }^{\circ} \mathrm{C}$; MOHAPATRA \& RAO (2005) encontraram, para o trigo parbolizado, 37,013 kJ mol ${ }^{-1}$, para a temperatura de secagem entre 40 e $60{ }^{\circ} \mathrm{C}$.

Ainda na Tabela 3, verifica-se que o coeficiente " $n$ " do modelo de Page, que reflete a resistência interna do produto à secagem, aumentou linearmente com a elevação da temperatura de secagem, podendo ser estimado, para a faixa de temperatura estudada pela eq.(18):

$$
\mathrm{n}=0,4916+0,0052 \mathrm{~T} \quad \mathrm{R}^{2}=94,98 \%
$$

em que,

$\mathrm{T}$ - temperatura do ar de secagem, ${ }^{\circ} \mathrm{C}$.

Na Figura 3, são apresentados os teores de água observados e estimados pelo modelo de Page, utilizando a representação de Arrhenius, para a secagem do feijão em camada fina, nas diversas condições de temperatura e umidade relativa do ar de $40 \pm 2 \%$.

Observa-se, na Figura 3, que, com o aumento da temperatura do ar de secagem, ocorre maior taxa de remoção de água do produto, como observado por diversos pesquisadores para inúmeros produtos agrícolas (OZDEMIR \& DERVES, 1999; BASUNIA \& ABE, 2001; YALDIZ, et al., 2001; AZZOUZ et al., 2002; KAYMAK-ERTEKIN, 2002; AKPINAR et al., 2003; LAHSASNI et al., 2004; ERTEKIN \& YALDIZ, 2004; MOHAPATRA \& RAO, 2005). O tempo necessário para que o feijão atingisse o teor de água de $12 \%$ b.u. foi de 20,0; 8,0 e 5,3 horas, para as temperaturas de 35; 45 e $55^{\circ} \mathrm{C}$, respectivamente.

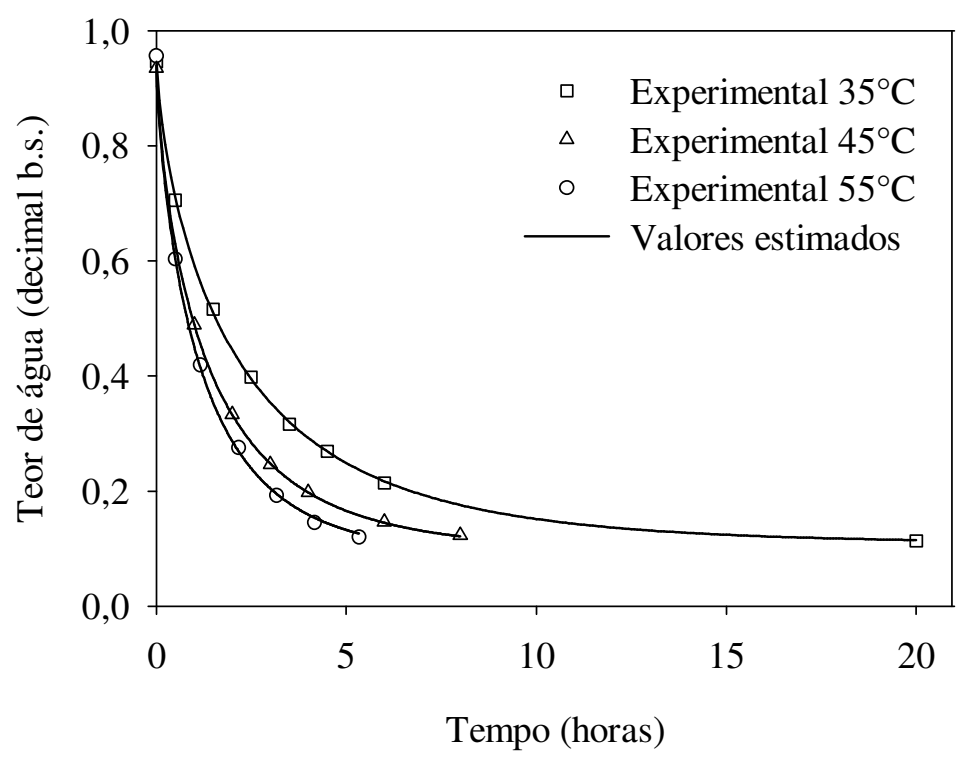

FIGURA 3. Teores de água experimentais e estimados pelo modelo de Page para a secagem do feijão em camada fina, nas diversas condições de temperatura e umidade relativa do ar de $40 \pm 2 \%$.

\section{CONCLUSÕES}

Baseando-se em parâmetros estatísticos, os modelos Page, Midilli, Henderson e Pabis modificado, Dois termos e Aproximação da difusão, dentre a série testada, são os que melhor representam o fenômeno de secagem do feijão, tendo sido o tradicional modelo de Page selecionado para descrever a cinética de secagem do feijão em camada fina. A relação entre a 
constante de secagem $\mathrm{k}$ desse modelo e a temperatura do ar pode ser descrita pela relação de Arrhenius, apresentando energia de ativação de $10,08 \mathrm{~kJ} \mathrm{~mol}^{-1}$. A constante $\mathrm{n}$ de secagem, ajustada pelo modelo de Page, variou linearmente com a temperatura do ar de secagem.O tempo necessário para a secagem do feijão até o teor de água de 0,14 (b.s.) foi de 20,0; 8,0 e 5,3 horas, para as temperaturas de 35,45 e $55^{\circ} \mathrm{C}$, respectivamente.

\section{REFERÊNCIAS}

AFONSO JÚNIOR, P.C.; CORRÊA, P.C. Comparação de modelos matemáticos para descrição da cinética de secagem em camada fina de sementes de feijão. Revista Brasileira de Engenharia Agrícola e Ambiental, Campina Grande, v.3, n.3, p.349-53, 1999.

AKPINAR, E.K.; BICER, Y.; YILDIZ, C. Thin layer drying of red pepper. Journal of Food Engineering, London, v.59, n.1, p.99-104, 2003.

AZZOUZ, S.; GUIZANI, A.; JOMAA, W.; BELGHITH, A. Moisture diffusivity and drying kinetic equation of convective drying of grapes. Journal of Food Engineering, London, v.55, n.4, p.323-30, 2002.

BABALIS, S.J.; BELESSIOTIS, V.G. Influence of the drying conditions on the drying constants and moisture diffusivity during the thin-layer drying of figs. Journal of Food Engineering, London, v.65, n.3, p.449-58, 2004.

BASUNIA, M.A.; ABE, T. Moisture desorption isotherms of medium-grain rough rice. Journal of Stored Products Research, London, v.37, n.2, p.205-19, 2001.

BERBERT, P.A.; QUEIROZ, D.M.; SILVA, J.S.; PINHEIRO FILHO, J.B. Simulation of coffe drying in a fixed bed with periodic airflow reversal. Journal of Agricultural Engineering Research, London, v.60, n.3, p.167-73, 1995.

BROOKER, D.B.; BAKKER-ARKEMA, F.W.; HALL, C.W. Drying and storage of grains and oilseeds. Westport: The AVI Publishing Company, 1992. 450 p.

CHRIST, D. Curvas de equilíbrio higroscópico e de secagem da canola (Brassica napus L. var. oleifera) e efeito da temperatura e da umidade relativa do ar de secagem sobre a qualidade das sementes. 1996. 50 f. Dissertação (Mestrado em Pré-processamento de Produtos Agrícolas) Universidade Federal de Viçosa, Viçosa, 1996.

DOYMAZ, I.; PALA, M. The thin-layer drying characteristics of corn. Journal of Food Engineering, London, v.60, n.2, p.125-30, 2003.

ERTEKIN, C.; YALDIZ, O. Drying of eggplant and selection of a suitable thin layer drying model. Journal of Food Engineering, London, v.63, n.3, p.349-59, 2004.

HALL, C.W. Drying and storage of agricultural crops. Westport: AVI, 1980. 381 p.

INCROPERA, F.P.; DEWITT, D.P. Fundamentos de transferência de calor e de massa. Rio de Janeiro: Editora Guanabara Koogan, 1992. 380 p.

KAYMAK-ERTEKIN, F. Drying and rehydrating kinetics of green and red peppers. Journal of Food Science, N. La Salle St, v.67, n.1, p.168-75, 2002.

KREYGER, J. Pratical observations on the drying of seed. Seed Science and Technology, Zürich, v.1., p.645-670, 1973.

KROKIDA, M.K.; FOUNDOUKIDIS, E.; MAROULIS, Z. Drying constant: literature data compilation for foodstuffs. Journal of Food Engineering, London, v.61, n.3, p.321-30, 2004.

KROKIDA, M.K.; KARATHANOS, V.T.; MAROULIS, Z.B.; MARINOS-KOURIS, D. Drying kinetics of some vegetables. Journal of Food Engineering, London, v.59, n.4, p.391-403, 2003. 
LAHSASNI, S.; KOUHILA, M.; MAHROUZ, M.; JAOUHARI, J.T. Drying kinetcs of prickly pear fruit (Opuntia ficus indica). Journal of Food Engineering, London, v.61, n.2, p.173-9, 2004.

MADAMBA, P.S.; DRISCOLL, R.H.; BUCKLE, K.A. Thin-layer drying characteristcs of garlic slices. Journal of Food Engineering, London, v.29, n.1, p.75-97, 1996.

MOHAPATRA, D.; RAO, P.S. A thin layer drying model of parboiled wheat. Journal of Food Engineering, London, v.66, n.4, p.513-18, 2005.

OZDEMIR, M.; DEVRES, Y.O. The thin layer drying characteristics of hazelnuts during roasting. Journal of Food Engineering, London, v.42, n.4, p.225-33, 1999.

PRADO, M.E.T.; ALONSO, L.F.T.; PARK, K.J. Shrinkage of dates (Phoenix Dacyilyfera L.) during drying. Drying Technology, New York, v.18, n.2, p.295-310, 2000.

RAMESH, M.N. Moisture transfer properties of cooked rice during drying. Journal of Food Science, N. La Salle St., v.36, n.2, p.245-55, 2003.

RAMOS, A.M.; PEREIRA, J.A.M.; QUEIROZ, D.M. Equações de secagem de trigo em camada fina e determinação dos coeficientes de difusão líquida. Revista Brasileira de Armazenamento, Viçosa, v.18-19, n.1-2, p.43-7, 1993/1994.

RAMOS, I.N.; BRANDÃO, T.R.S.; SILVA, C.L.M. Integrated approach on solar drying, pilot convective drying and microstructural changes. Journal of Food Engineering, London, v.67, n.1-2, p.195-203, 2005.

RAMOS, I.N.; SILVA, C.L.M.; SERENO A.M.; AGUILERA, J.M. Quantification of microstructural changes during first stage air drying of grape tissue. Journal of Food Engineering, London, v.62, n.2, p.159-64, 2004.

SUN, D.W.; WOODS, J.L. Low temperature moisture transfer characteristcs of wheat in thin layers. Transactions of ASAE, St. Joseph, v.37, n.4, p.1919-26, 1994.

YALDIZ, O.; ERTEKIN, C.; UZUN, H.I. Mathematical modeling of thin layer solar drying of sultana grapes. Energy, London, v.26, n.5, p.457-65, 2001.

ZOGZAS, N.P.; MAROULIS, Z.B.; MARINOS-KOURIS, D. Moisture diffusivity data compilation in foodstuffs. Drying Technology, New York, v.14, n.10, p.2225-53, 1996. 